\title{
ÚNG DỤNG PHƯƠNG PHÁP ROBUST BÌNH SAI VÀ PHÂN TÍCH LƯỚI KHÔNG GIAN MẠT ĐẤT - GPS
}

\author{
HOÀNG NGỌC HÀ, LƯU ANH TUẤN \\ Truòng Đại học Mỏ - Địa chất
}

\section{Tóm tắt:}

Phwơng pháp bình sai theo nguyên lý số bình phương nhỏ nhất chỉ phù hợp khi trị đo có chứa sai số ngẫu nhiên, tuy nhiên trong thực tế ngoài sai số ngẫu nhiên các trị đo còn chứa cả sai số thô. Hiện nay, một trong nhũng phương pháp hiệu quả xủ lý sai số thô là phuơng pháp ước lượng vũng (Robust estimation). Một đặc điểm quan trọng của phương pháp ước lượng vũng là kết quả của mỗi phuơng pháp wớc lương vũng phu thuộc việc lụa chọn hàm trọng số của nó, do đó bài báo úng dụng phưong pháp wớc luợng vũng với hàm trọng số hợp lý để bình sai và phân tích luới không gian mặt đất-GPS.

\section{1. Đặt vấn đề}

Công nghệ GPS kết hợp công nghệ đo đạc truyền thống tạo thành mạng lưới hỗn hợp trị đo mặt đất - GPS đã và đang là giải pháp hữu hiệu trong công tác xây dựng lưới trắc địa ở Việt Nam. Đã có một số nghiên cứu ứng dụng phương pháp ước lượng vững để phát hiện trị do có chứa sai số thô trong lưới trắc địa, tuy nhiên, vấn đề xử lý, phân tích chất lượng lưới trắc địa không gian có nhiều loại trị đo khác nhau khi trị đo có chứa sai số thô ở Việt Nam vẫn là vấn đề mới mang tính thời sự. Do đó, trong bài báo này chúng tôi tập trung sử dụng phương pháp Robust để tìm kiếm, xử lý và phân tích lưới trắc địa không gian mặt đất - GIS.

Lý thuyết cơ bản của ước lượng vững là trong trường hợp sai số thô không thể tránh được, chọn phương pháp ước lượng phù hợp để cho trị ước lượng của các tham số không bị ảnh hưởng hoặc bị ảnh hưởng nhỏ nhất. Tuy nhiên, việc lựa chọn hàm trọng số trong phương pháp ước lượng vững khác nhau sẽ cho kết quả khác nhau. Do đó, để xử lý và phân tích chất lượng lưới trắc địa có nhiều loại trị đo như lưới trắc địa không gian mặt đất - GPS chúng tôi đề xuất sử dụng hàm trọng số hợp lý trong ước lượng vững.

\section{Giải quyết vấn đề}

\subsection{Phương pháp ước lự̛ng vĩng}

Giả thiết trị đo độc lập $L_{n x l}$, vector tham số cần xác định là

$$
V_{n x 1}=A_{n x t} \hat{X}_{n \mathrm{x}}+L_{n x \mathrm{l}}=\left[\begin{array}{c}
a_{1} \\
a_{2} \\
\ldots \\
a_{n}
\end{array}\right]_{n x t}\left[\begin{array}{c}
\hat{X}_{1} \\
\hat{X}_{2} \\
\ldots \\
\hat{X}_{t}
\end{array}\right]_{n=1}+\left[\begin{array}{c}
l_{1} \\
l_{2} \\
\ldots \\
l_{n}
\end{array}\right]
$$

Trong đó: $A_{n x n}$ là ma trận hệ số, $a_{i}$ là vector hệ số, $L_{n x l}$ là ma trận số hạng tự do.

Phương trình $(1)$, hàm số $\rho\left(l_{i}, \hat{X}\right)$ của ước lượng $\mathrm{M}$ có dạng [5]:

$$
\rho\left(l_{i}, \hat{X}\right)=\rho\left(v_{i}\right)
$$

Do các trị đo không cùng độ chính xác, ma trận trọng số $\mathrm{P}$ có dạng

$$
P_{n x n}=\left(\begin{array}{lllll}
p_{1} & & & & \\
& p_{2} & & & \\
& & \cdots & & \\
& & & \cdots & \\
& & & p_{n}
\end{array}\right)_{n x n}
$$

Khi đó hàm $p$ có dạng

$$
\sum_{i=1}^{n} p_{i} \rho\left(v_{i}\right)=\sum_{i=1}^{n} p_{i} \rho\left(a_{i} \hat{X}+l_{i}\right)
$$

Lấy đạo hàm của biểu thức (4) đối với X, ký

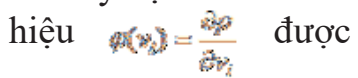

Ngày nhận bài: 11/1/2019, ngày chuyển phản biện: 21/1/2019, ngày chấp nhận phản biện: 30/1/2019, ngày chấp nhận đăng: 11/2/2019 


$$
\sum_{i=1}^{n} p_{i} \varphi\left(v_{i}\right) a_{i}=0
$$

Đặt $\bar{p}_{i}=p_{i} w_{i}, w_{i}=\frac{\varphi\left(v_{i}\right)}{v_{i}}$, được

$$
\sum_{i=1}^{n} a_{i}^{T} \bar{p} v_{i}=0
$$

hoặc

$$
A^{T} P V=0
$$

Thay $V=A \hat{X}+L, \quad$ vào (7) được hệ phương trình chuẩn của ước lượng $\mathrm{M}$ là

$$
A^{T} \bar{P} A \hat{X}+A^{T} \bar{P} L=0
$$

Trong đó: $\bar{P}$ là ma trận trọng số tương đương, $\bar{p}_{i}$ là phần tử trọng số tương đương, $w_{i}$ là hệ số trọng số.

Tham số $\hat{X}$ của ước lượng vững $M$ được xác định

$$
\hat{X}=\left(A^{T} \bar{P} A\right)^{-1} x A^{T} \bar{P} L
$$

\subsection{Lự chọn hàm trọng số ước lượng vũng}

$$
\begin{aligned}
& \left\{\begin{array}{l}
\mathrm{w}_{i}=1 \\
\mathrm{w}_{i}=\frac{c}{\mid \bar{v}_{i}} ;\left|\bar{v}_{i}\right|=\frac{v_{i}}{\sigma_{v}} ; \bar{p}_{i}=p_{i} \mathrm{w}_{i} ;
\end{array}\right. \\
& \mathrm{w}_{i l}=\mathrm{w}_{i}=1 \text {; } \\
& \mathrm{w}_{i j}=\frac{c}{\left|\bar{v}_{i}\right|} ;\left|\bar{v}_{i}\right|=\frac{v_{i}}{\sigma_{v_{j}}} ; \mathrm{w}_{b j}=\frac{c}{\left|\bar{v}_{j}\right|} ;\left|\bar{v}_{j}\right|=\frac{v_{j}}{\sigma_{v_{j}}} ; \bar{p}_{i j}=p_{i j} \mathrm{w}_{i j} ;
\end{aligned}
$$

Trong đó, $\mathrm{c}$ là hằng số và được chọn $\mathrm{c}=1.5$.

\subsection{Tính toán thục nghiệm}

Bài báo sử dụng lưới trắc địa được xây dựng trong khu vực Sân bay Nội Bài, TP Hà Nội. Lưới gồm 4 điểm bao gồm 1 điểm gốc $\mathrm{NB} 1$ và 3 điểm cần xác định NB2, NB3, NB4 được đo bằng máy đo GPS và 4 cạnh được đo bằng máy đo toàn đạc điện tử có độ chính xác đo cạnh là $(2+2$ ppm).
Lưới GPS kết hợp trị đo cạnh mặt đất được bình sai trong hệ tọa độ địa diện. (Xem bảng 1, 2, 3, 4, 5, biểu đồ 1,2)

\section{Nhận xét:}

Kết quả tính toán thực nghiệm phương pháp ước lượng vững thể hiện ở bảng 4 , bảng 5 và biểu đồ 1 , biểu đồ 2 cho thấy:

- Các trị đo 1 (cạnh NB3 - NB4) và trị đo 5 $\left(\Delta X_{\mathrm{NB} 3-\mathrm{NB} 2}\right)$ được gán sai số thô đều được phát hiện chính xác

- Độ lớn của sai số thô xác định được xấp xỉ bằng giá trị sai số thô đưa vào mô hình thực nghiệm.

- Phương pháp này cho phép tìm kiếm trị đo thô từ đó giúp người xử lý số liệu có thể tạo ra dữ liệu đo "sạch" và bình sai theo phương pháp thông thường.

\section{Kết luận}

Phương pháp Robust được nghiên cứu trong bài toán này với lựa chọn hàm trọng số hợp lý để

\begin{tabular}{|c|c|c|c|c|}
\hline \multirow[t]{2}{*}{ STT } & \multicolumn{2}{|c|}{ Tên cạnh } & \multirow[t]{2}{*}{ Chiều dài $(\mathrm{m})$} & \multirow{2}{*}{$\begin{array}{l}\text { Gán sai số thô } \\
(\mathrm{cm})\end{array}$} \\
\hline & Điểmđầu & Điểmcuói & & \\
\hline 1 & NB1 & NB4 & 900.177 & \\
\hline 2 & NB4 & NB2 & 597.890 & \\
\hline 3 & NB2 & NB1 & 717.567 & \\
\hline 4 & NB3 & NB4 & 667.755 & +10 \\
\hline
\end{tabular}
tìm kiếm trị đo chứa sai số thô, xử lý và phân tích chất lượng lưới trắc địa không gian mặt đất - GPS cho kết quả đáng tin cậy. $\bigcirc$

\section{Tài liệu tham khảo}

[1]. Hoàng Ngọc Hà, 2006. Bình sai tính toán lưới trắc địa và GPS, nhà xuất bản khoa học kỹ thuật, Hà Nội.

[2]. Huber, P.J., 1964. Robust estimation of a location parameter. Annals of Mathematical Statistics, 35: 73-101.

\section{Bảng 1: Trị đo cạnh mặt đất}


Bảng 2: Trị đo GPS $\triangle X, \Delta Y, \Delta Z$ và ma trận hiệp phưong sai trong hệ địa tâm

\begin{tabular}{|c|c|c|c|c|c|}
\hline STT & THÔNG TIN & ĐIÉM ĐÁU & & ĐIÉM CUÔI & GHI CHÚ \\
\hline \multirow{5}{*}{1} & BASELINE & NBl & & NB2 & \\
\hline & $\Delta \mathrm{X} \Delta \mathrm{Y} \Delta \mathrm{Z}$ (Global) & $608.209 \mathrm{~m}$ & $276.986 \mathrm{~m}$ & $-261.296 \mathrm{~m}$ & \\
\hline & \multirow{3}{*}{ HPS } & 0.00000033 & -0.00000025 & -0.00000009 & \\
\hline & & & 0.00000116 & 0.00000039 & \\
\hline & & & & 0.00000035 & \\
\hline \multirow{5}{*}{2} & BASELINE & NBl & & NB4 & \\
\hline & $\Delta \mathrm{X} \Delta \mathrm{Y} \Delta \mathrm{Z}($ Global $)$ & $849.586 \mathrm{~m}$ & $132.541 \mathrm{~m}$ & $266.374 \mathrm{~m}$ & \\
\hline & \multirow{3}{*}{ HPS } & 0.00000083 & -0.00000068 & -0.00000027 & \\
\hline & & & 0.00000325 & 0.00000121 & \\
\hline & & & & 0.00000101 & \\
\hline \multirow{5}{*}{3} & BASELINE & NBl & & NB3 & \\
\hline & $\Delta \mathrm{X} \Delta \mathrm{Y} \Delta \mathrm{Z}$ (Global) & $1375.445 \mathrm{~m}$ & $405.586 \mathrm{~m}$ & $-41.548 \mathrm{~m}$ & \\
\hline & \multirow{3}{*}{ HPS } & 0.000000315 & -0.000000246 & -0.000000096 & \\
\hline & & & 0.000001103 & 0.000000364 & \\
\hline & & & & 0.000000330 & \\
\hline \multirow{5}{*}{4} & BASELINE & NB2 & & NB4 & \\
\hline & $\Delta \mathrm{X} \Delta \mathrm{Y} \Delta \mathrm{Z}($ Global $)$ & $241.374 \mathrm{~m}$ & $-144.445 \mathrm{~m}$ & $527.671 \mathrm{~m}$ & \\
\hline & \multirow{3}{*}{ HPS } & $8.047 \mathrm{E}-07$ & $-6.557 \mathrm{E}-07$ & -0.000000263 & \\
\hline & & & $3.1558 \mathrm{E}-06$ & $1.1713 \mathrm{E}-06$ & \\
\hline & & & & $9.804 \mathrm{E}-07$ & \\
\hline \multirow{5}{*}{5} & BASELINE & NB3 & & NB4 & \\
\hline & $\Delta \mathrm{X} \Delta \mathrm{Y} \Delta \mathrm{Z}$ (Global) & $-525.864 \mathrm{~m}$ & $-273.045 \mathrm{~m}$ & $307.924 \mathrm{~m}$ & \\
\hline & \multirow{3}{*}{ HPS } & $8.094 \mathrm{E}-07$ & $-6.595 \mathrm{E}-07$ & $-2.645 \mathrm{E}-07$ & \\
\hline & & & $3.1741 \mathrm{E}-06$ & $1.1781 \mathrm{E}-06$ & \\
\hline & & & & $9.861 \mathrm{E}-07$ & \\
\hline \multirow{5}{*}{6} & BASELINE & NB3 & & NB2 & \\
\hline & $\Delta \mathrm{X} \Delta \mathrm{Y} \Delta \mathrm{Z}($ Global $)$ & $-767.238 \mathrm{~m}$ & $-128.600 \mathrm{~m}$ & $-219.746 \mathrm{~m}$ & \\
\hline & \multirow{3}{*}{ HPS } & $5.017 \mathrm{E}-07$ & $-3.573 \mathrm{E}-07$ & $-1.205 \mathrm{E}-07$ & \\
\hline & & & $1.6939 \mathrm{E}-06$ & $5.607 \mathrm{E}-07$ & \\
\hline & & & & $5.099 \mathrm{E}-07$ & \\
\hline
\end{tabular}

Bảng 4: Kết quả độ lệch số hiệu chỉnh trị đo GPS khi dãy trị đo chứa sai số thô và khi dãy trị đo không chứa sai số thô

\begin{tabular}{|c|c|c|c|c|c|c|}
\hline \multirow{2}{*}{ STT } & \multicolumn{2}{|c|}{ Tên Baseline } & \multicolumn{3}{c|}{ Độ lệch số hiệu chinh } & \multirow{2}{*}{$\begin{array}{c}\text { Giá trị sai số thô } \\
\text { (mm) }\end{array}$} \\
\cline { 2 - 6 } & Điểmđầu & Điểmcuối & $\mathrm{dV} \Delta \mathrm{x}(\mathrm{mm})$ & $\mathrm{dV} \Delta \mathrm{y}(\mathrm{mm})$ & $\mathrm{dV} \Delta \mathrm{z}(\mathrm{mm})$ & \\
\hline 1 & NB3 & NB2 & 99.95 & 4.00 & 0.00 & $\Delta \mathrm{x}+100$ \\
\hline 2 & NB2 & NB4 & 0.01 & 0.01 & 1.65 & \\
\hline 3 & NB3 & NB4 & 0.03 & 3.99 & 1.66 & \\
\hline 4 & NB1 & NB2 & 0.00 & 2.00 & 0.90 & \\
\hline 5 & NB1 & NB4 & 0.01 & 1.99 & 0.75 & \\
\hline 6 & NB1 & NB3 & 0.05 & 2.00 & 0.91 & \\
\hline
\end{tabular}


Bảng 3: Trị đo GPS $\Delta x, \Delta y, \Delta z$ và ma trận hiệp phuong sai trong hệ địa diện

\begin{tabular}{|c|c|c|c|c|c|}
\hline STT & THÖNG TIN & ĐIÉM ĐÁU & & ĐIÉM CUÓI & Gánsaisốthô (cm) \\
\hline \multirow{5}{*}{1} & BASELINE & NBl & & NB2 & \multirow{5}{*}{$\Delta x+10$} \\
\hline & $\Delta x \Delta y \Delta z$ & $-280.172 \mathrm{~m}$ & $-660.620 m$ & $-0.354 \mathrm{~m}$ & \\
\hline & \multirow{3}{*}{ HPS } & $1.954 \mathrm{E}-07$ & $-1.662 \mathrm{E}-08$ & $-1.801 \mathrm{E}-09$ & \\
\hline & & & $2.605 \mathrm{E}-07$ & $-1.102 E-08$ & \\
\hline & & & & 1.384E-06 & \\
\hline \multirow{5}{*}{2} & BASELINE & NB1 & & NB4 & \\
\hline & $\Delta \mathrm{x} \Delta \mathrm{y} \Delta \mathrm{z}$ & $285.797 \mathrm{~m}$ & $-853.603 \mathrm{~m}$ & $-0.102 \mathrm{~m}$ & \\
\hline & \multirow{3}{*}{ HPS } & $4.913 \mathrm{E}-07$ & $-4.504 \mathrm{E}-08$ & $9.774 \mathrm{E}-08$ & \\
\hline & & & $6.531 \mathrm{E}-07$ & $-7.552 \mathrm{E}-08$ & \\
\hline & & & & $3.946 \mathrm{E}-06$ & \\
\hline \multirow{5}{*}{3} & BASELINE & NBl & & NB3 & \\
\hline & $\Delta \mathrm{x} \Delta \mathrm{y} \Delta \mathrm{z}$ & $-44.575 \mathrm{~m}$ & $-1433.907 \mathrm{~m}$ & $0.010 \mathrm{~m}$ & \\
\hline & \multirow{3}{*}{ HPS } & $1.865 \mathrm{E}-07$ & $-7.389 \mathrm{E}-09$ & $-6.914 \mathrm{E}-09$ & \\
\hline & & & $2.445 \mathrm{E}-07$ & $7.000 \mathrm{E}-10$ & \\
\hline & & & & $1.317 \mathrm{E}-06$ & \\
\hline \multirow{5}{*}{4} & BASELINE & NB2 & & NB4 & \\
\hline & $\Delta x \Delta y \Delta z$ & $565.969 \mathrm{~m}$ & $-192.981 \mathrm{~m}$ & $0.253 \mathrm{~m}$ & \\
\hline & \multirow{3}{*}{ HPS } & $4.805 \mathrm{E}-07$ & $-4.338 \mathrm{E}-08$ & $8.961 \mathrm{E}-08$ & \\
\hline & & & $6.291 \mathrm{E}-07$ & $-7.631 \mathrm{E}-08$ & \\
\hline & & & & $3.830 \mathrm{E}-06$ & \\
\hline \multirow{5}{*}{5} & BASELINE & NB3 & & NB4 & \\
\hline & $\Delta \mathrm{x} \Delta \mathrm{y} \Delta \mathrm{z}$ & $330.373 \mathrm{~m}$ & $580.308 \mathrm{~m}$ & $-0.110 \mathrm{~m}$ & \\
\hline & \multirow{3}{*}{ HPS } & $4.840 \mathrm{E}-07$ & $-4.592 \mathrm{E}-08$ & $9.671 \mathrm{E}-08$ & \\
\hline & & & $6.391 \mathrm{E}-07$ & $-7.729 \mathrm{E}-08$ & \\
\hline & & & & $3.847 \mathrm{E}-06$ & \\
\hline \multirow{5}{*}{6} & BASELINE & NB3 & & NB2 & \\
\hline & $\Delta \mathrm{x} \Delta \mathrm{y} \Delta \mathrm{z}$ & $-235.596 \mathrm{~m}$ & $773.286 \mathrm{~m}$ & $-0.363 \mathrm{~m}$ & \\
\hline & \multirow{3}{*}{ HPS } & $1.934 \mathrm{E}-07$ & $-2.061 \mathrm{E}-08$ & $-7.110 \mathrm{E}-09$ & \\
\hline & & & $2.659 \mathrm{E}-07$ & $-1.154 \mathrm{E}-08$ & \\
\hline & & & & $1.326 \mathrm{E}-06$ & \\
\hline
\end{tabular}

Bảng 5: Kết quả độ lệch số hiệu chỉnh trị đo mặt đất khi dãy trị đo chưa sai số thô và khi dãy trị đo không chứa sai số thô

\begin{tabular}{|c|c|c|c|c|}
\hline \multirow{2}{*}{ STT } & \multicolumn{2}{|c|}{ Tên cạnh } & Độ lệch số hiệu chỉnh & $\begin{array}{c}\text { Giá trị sai số thô } \\
\text { (mm) }\end{array}$ \\
\cline { 2 - 5 } & Điểm đầu & Điểm cuối & dVS(mm) & \\
\hline 1 & NB1 & NB4 & 96.55 & $\mathrm{~S}+100$ \\
\hline 2 & NB4 & NB2 & 0.02 & \\
\hline 3 & NB2 & NB1 & 1.84 & \\
\hline 4 & NB3 & NB4 & 1.88 & \\
\hline
\end{tabular}


Biểu đồ 1: Kết quả độ lệch số hiệu chỉnh

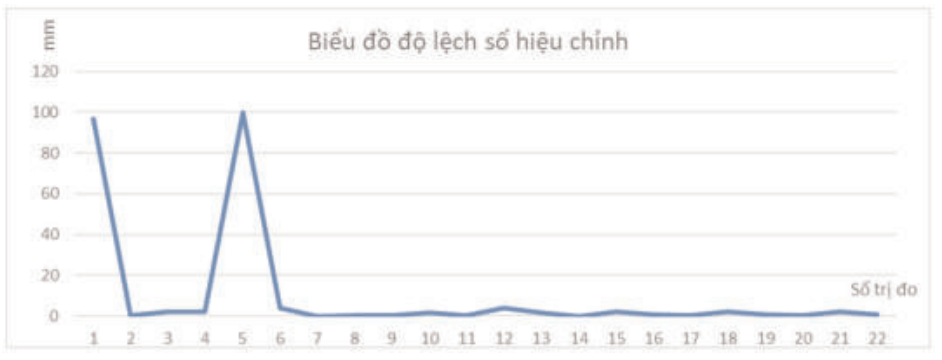

Biểu đồ 2: Phát hiện trị đo chứa sai số thô

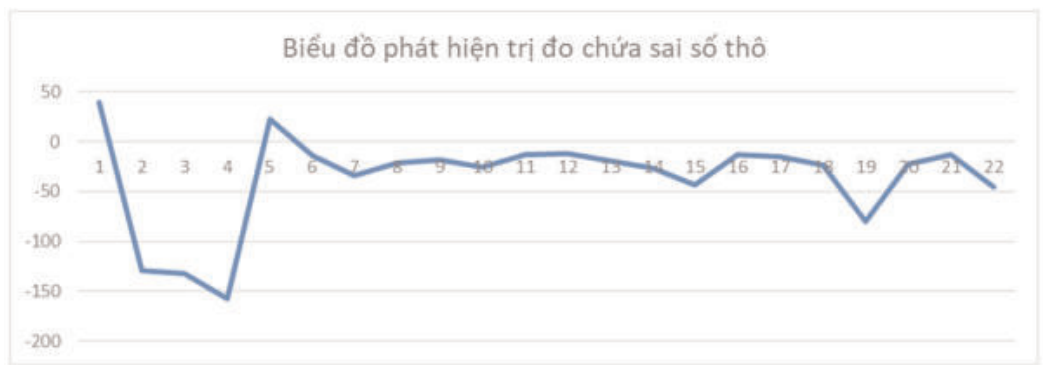

[3]. Huber, P.J., 1972. Robust statistics - A of geodetic network", GMMT2016 review. Annals of Mathematical Statistics, 43: International symposium on Geo-Spatial and 1041-1067.

[4]. Huber, P.J., 1981. Robust Statistics. John Wiley \& Sons, New York.

[5]. Wang Xinhou, Tao Benzao, QiuWeining, Yao Yibin. Advanced surveying adjustment; PGS. TS Phan Văn Hiến dịch.

[6]. Anh Tuan Luu (2016),“Application of correlation robust estimation in the adjustment Mobile mapping technologies and summer school for Mobile mapping technology.

[7]. Anh Tuan Luu, Ngoc Giang Le (2016), "Application of weighting robust estimation in geodetic networks", GMMT2016 - International symposium on Geo-Spatial and Mobile mapping technologies and summer school for Mobile mapping technology. $\bigcirc$

\section{Summary}

Application of Robust method in adjustment and analysis for network space combain terrain and global positoning system.

Hoang Ngoc Ha, LuuAnh Tuan

\section{Hanoi University of Mining and Geology}

Adjustment base on least square estimation is optimal when measurements only contain random tolerances, but in fact, the measurementscontain outliers. Currently, one of the most effective methods of handling raw errors is Robust estimation. An important feature of the robust estimation method is the result of each robust estimation method depending on its weighting function selection. The contents of the article refer to the application of estimation method in adjustment and analysis for Network space combain terrain and Global Positioning System. $\bigcirc$ 